\title{
Correction to: Supporting Mental Health in Young Refugees: A Resilience Perspective
}

Sofie Vindevogel and An Verelst

\section{Correction to: Chapter 4 in: S. J. Song, P. Ventevogel (eds.), Child, Adolescent and Family Refugee Mental Health, https://doi.org/10.1007/978-3-030-45278-0_4}

The spelling of the author name Sophie Vindevogel was incorrect in the original publication. The name should read as Sofie Vindevogel. The correction has been updated in chapter.

The updated version of this chapter can be found at https://doi.org/10.1007/978-3-030-45278-0_4 